\title{
The simulation and analysis of a U-shaped slide valve based on Fluent
}

\author{
He Pengcheng ${ }^{1}$ \\ ${ }^{1}$ Faculty of Mechanical Engineering, ChangdeTechnicianCollege, \\ Changde, Hunan 415000, China
}

\begin{abstract}
The numerical simulation of a 3-D model of the internal flow field for a U-shaped slide valve has been analyzed in this paper. The impact of different opening degree on the pressure and velocity were analyzed. The results show that in the U-shaped groove, the pressure changes rapidly, and in the outlet oil chamber, the vortex was appeared. According to the expression of the flow coefficient, the flow characteristic was predicted by CFD, the conclusions show that with the increase of opening degree, the flow gradually increases, and the flow coefficient was not a stable value.
\end{abstract}

\section{Introduction}

Hydraulic valve is one of the fundamental components used in hydraulic system. It functions to control pressure, flow rate and operating direction of a execute component. The internal structure of hydraulic valve is more complex.

In recent years, with the continuous development of computer science and technology, computational fluid dynamics has been widely used, it is important to understand the working mechanism, improve design efficiency and structural optimization by using Fluent software.

Due to the fluid flow in the valve is more complex, it is necessary to research the law of flow which inside the hydraulic valve through the computer software-Fluent. To obtain the flow force of valve, Wang Guozhi[1] carried out the numerical simulation by CFD. Zhang Qinglan[2] and Zhang Jing[3] had been analyzed the threedimensional steady-state models of valve, and come to the conclusion that the along with the different opening of valve, the pressure and speed distribution present various pattern. Currently, the study of valve is mainly focused on the circular opening spool valve, and the research reports of non-circular opening spool valve are few.

This paper with U-shaped slide valve of non-circular opening as the object of the research, adopts the method of numerical simulation to study the law of internal flow which contains speed, pressure flow characteristics and flow coefficient. On this basis, CFD technology was applied to perform a three-dimensional simulation of the valve chamber flow field. The simulation can confirm the accuracy of the U-shaped slide valve and predict system parameters, provide for a reference of slide valve of the optimization design.

\section{Geometric model and calculation conditions}

The proposed U-shaped slide valve shown in Fig.1, where $A$ is the fluid inlet and B is the fluid outlet, $x$ is the valve opening degree of slide valve, the slide valve is composed of a spool valve, a static sleeve[4]. There are four Ushaped groove on each convex shoulder, the model of Ushaped groove shown in Fig.2, the movement of spool valve can form different valve opening degree and different orifice areas. The specific dimension parameters of slide valve in table 1 .

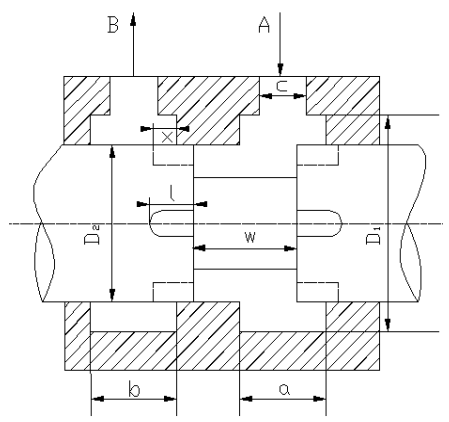

Figure 1. The structure of slide valve model

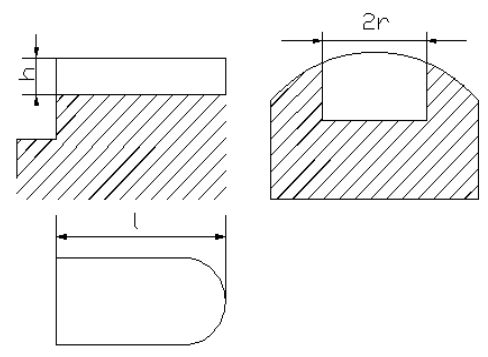

Figure 2. The schematic of U-shaped groove 
Table.1 The main parameters of the U-shaped slide valve $(\mathrm{mm})$

\begin{tabular}{|l|l|l|l|}
\hline$a$ & 10 & $w$ & 12 \\
\hline$b$ & 10 & $h$ & 2 \\
\hline$c$ & 6 & $r$ & 1 \\
\hline$D_{1}$ & 24 & $l$ & 5 \\
\hline$D_{2}$ & 18 & & \\
\hline
\end{tabular}

Based upon the above models, the results of the three dimensional modeling of slide valve and valve plug are established by using Pro/e software, the model is seen in Fig.3. Moving the spool valve, can get different opening degrees, in this paper, 10 different opening degrees of valve was calculated from $1 \mathrm{~mm}$ to $5 \mathrm{~mm}$.

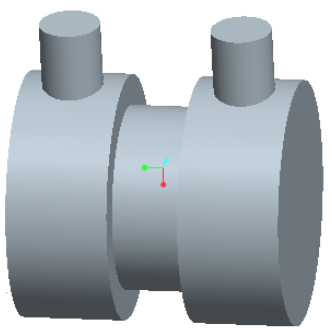

(a)The fluid region without valve plug

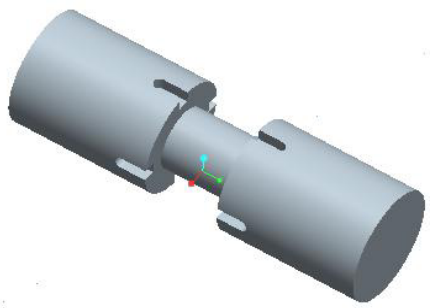

(b) Valve plug

Figure 3. The three-dimensional model

CFD analysis using ANSYS/FLUENT based simulation is applied to verify the models and study the internal flow characteristics. The mesh picture is shown in Fig.4, the opening degree of slide valve is $3 \mathrm{~mm}$, where $\mathrm{A}$ is the fluid inlet and $\mathrm{B}$ is the fluid outlet.

In order to solve the calculation problem, the following assumptions are made:

(1) The simulation fluid incompressible Newtonian

(2) Fluid medium is hydraulic oil which density is $860 \mathrm{~kg} / \mathrm{m} 3$, kinematic viscosity is $4 * 105 \mathrm{~m} 2 / \mathrm{s}$.

The inlet boundary condition is defined as pressure inlet, which the inlet pressure is $2 \mathrm{Mpa}$, in the computational domain of the outlet, the pressure outlet boundary condition is used which the out pressure is $0.5 \mathrm{Mpa}$.

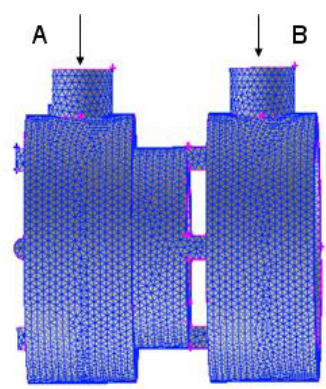

(a) The whole basin

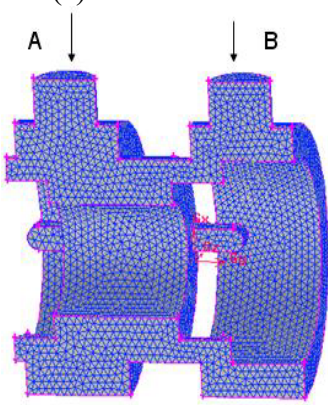

(b) The half cross section

Figure 4. The mesh of the spool valve when the opening is $3 \mathrm{~mm}$

\section{CFD analysis of pressure}

For the simulation of the internal characteristics of slide valve, two sets of constant opening degree, i.e, $x=1 \mathrm{~mm}$ and $x=3 \mathrm{~mm}$. The pressure cloud map in the symmetry plane of slide valve in the case of $1 \mathrm{~mm}, 3 \mathrm{~mm}$ opening degrees are shown in Fig.5(a) and (b), respectively, and the pressure contour map in the outlet of slide valve in the case of $1 \mathrm{~mm}, 3 \mathrm{~mm}$ opening degrees are shown in Fig.6(a) and (b). As seen in Fig.5, whether the opening degree $1 \mathrm{~mm}$ and $3 \mathrm{~mm}$, the trend of pressure distribution is the same, the pressure drop, mainly concentrated in the Ushaped groove, and in this area, the pressure gradient is larger than the rest of slide valve. With the increase of throttling groove opening degree, the pressure and the pressure drop will gradually increase. Comparison of $1 \mathrm{~mm}$ and $3 \mathrm{~mm}$ opening degree, opening degree of $1 \mathrm{~mm}$, the pressure drop of notch orifice is $0.35 \mathrm{Mpa}$, in the area of notch orifice, the maximum pressure is $0.85 \mathrm{Mpa}$. Opening degree of $3 \mathrm{~mm}$, the pressure drop and maximum pressure are $0.75 \mathrm{Mpa}$ and $1.3 \mathrm{Mpa}$, respectively. Due to the orifice area and flow capacity decrease, and this resulted the maximum pressure in throttling groove decrease by $0.45 \mathrm{Mpa}$.

As can be seen in Fig.6, in the case of $1 \mathrm{~mm}$, the pressure drop mainly concentrated at the location of outlet, and little change in other regions. 


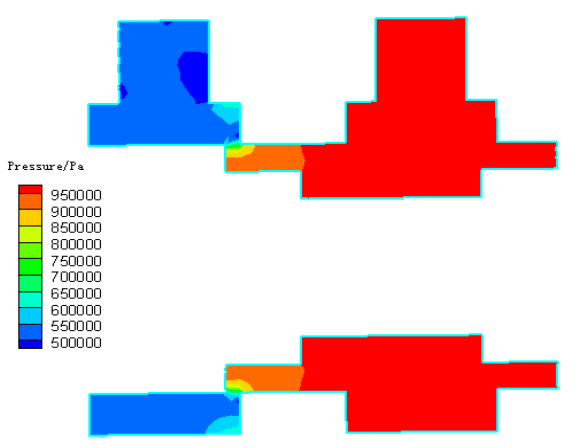

(a) Opening degree of $1 \mathrm{~mm}$

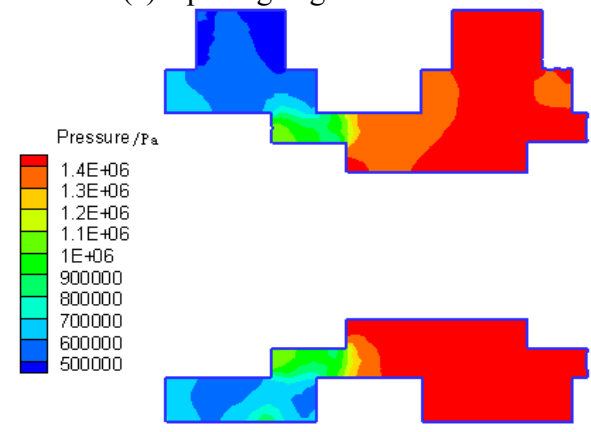

(b) Opening degree of $3 \mathrm{~mm}$

Figure 5. Comparison of pressure cloud map in different opening degrees

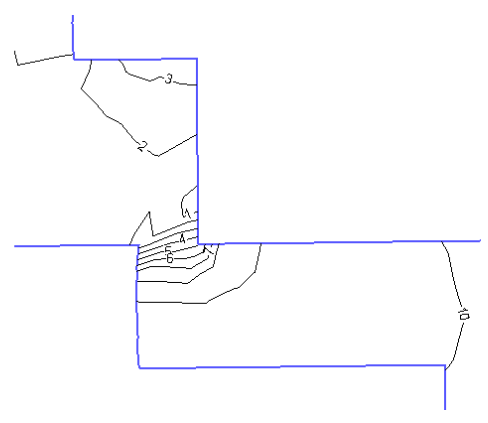

(a) Opening degree of $1 \mathrm{~mm}$

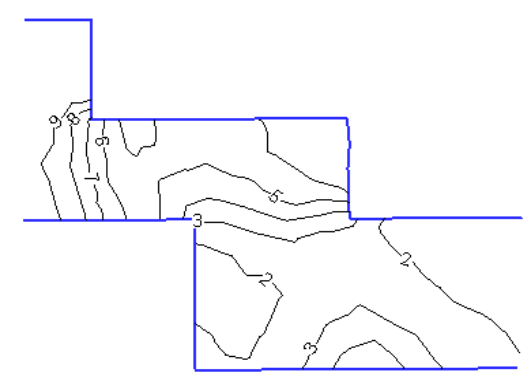

(b) Opening degree of $3 \mathrm{~mm}$

Figure 6. Comparison of pressure contour map in different opening degrees

\section{CFD analysis of flow rate}

In the above section, fluid pressure distributions in the chamber of slide valve was obtained, then using the same settings and calculations, the velocity distribution of slide valve was received, as can be seen from the velocity contours and vector shown in Fig.7 and Fig.8. In these two cases, the maximum velocity appeared in the outlet orifice, and there was an obvious low velocity on the left side of the wall which near the outlet oil chamber, where vortex was appeared. There was a significant difference between inlet oil chamber and outlet oil chamber, the velocity in the inlet oil chamber is very slow, and there was no drastic change of velocity, but in the outlet oil chamber, due to the effect of jet exit velocity, velocity has a dramatic change. The maximum velocity of the opening degree of $3 \mathrm{~mm}$ was $30 \mathrm{~m} / \mathrm{s}$, with the decrease of the opening degree of valve, the maximum velocity was reduced, and the maximum velocity of opening degree of $1 \mathrm{~mm}$ was $14 \mathrm{~m} / \mathrm{s}$. In that case, both of the low speed flow region and vortex region were greater than the opening degree of $3 \mathrm{~mm}$.
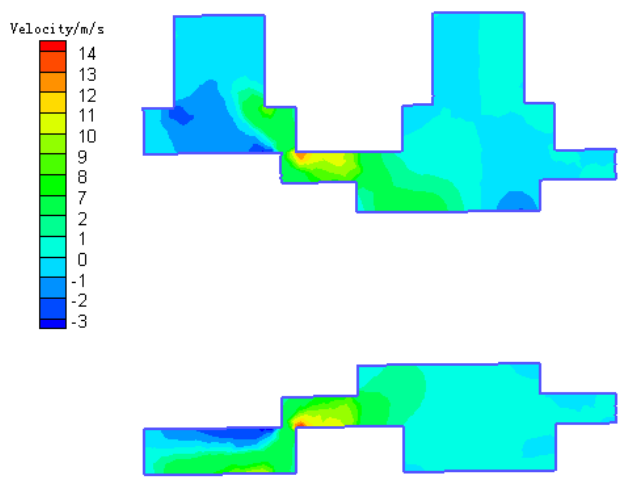

(a) The contours of Velocity
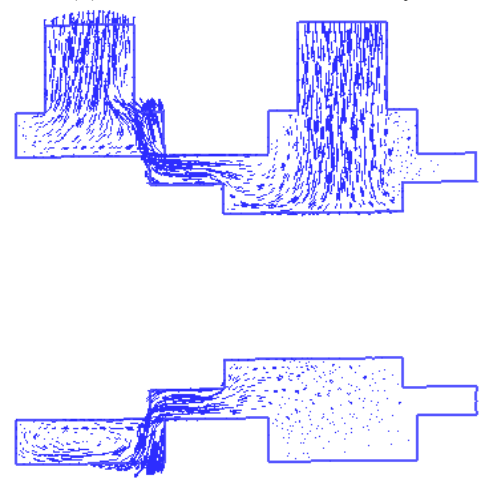

(b) The vector of Velocity

Figure 7. The velocity contours and vector distribution of the symmetry surfaceat opening degree of $1 \mathrm{~mm}$
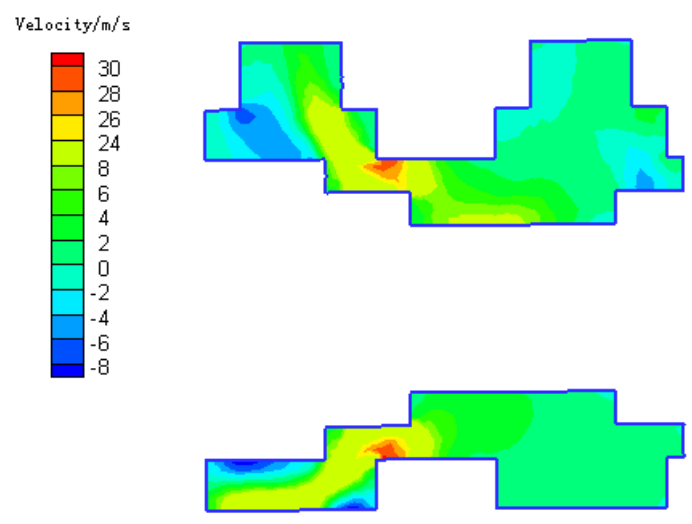

(a) The contours of Velocity 


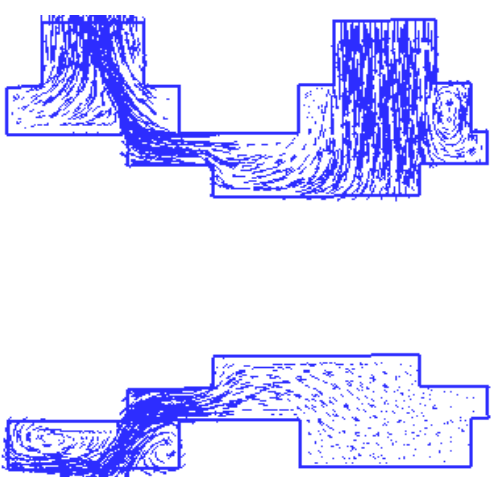

(b) The vector of Velocity

Figure 8. The velocity contours and vector distribution of the symmetry surface opening degree of $3 \mathrm{~mm}$

Valve port flow is an important parameter of the hydraulic system, flow characteristics was determined by the orifice area and flow coefficient[5]. According to the define of flow equation, the flow coefficient can be written as[6]

$$
C_{d}=\frac{q}{A} \sqrt{\frac{\rho}{2 \Delta p}}
$$

Where $\mathrm{Cd}$ is the flow coefficient, $\mathrm{q}$ is the flow, $\mathrm{A}$ is the orifice areas of valve, $\rho$ is the hydraulic oil density, $\Delta \mathrm{p}$ is the pressure difference, in this paper $\Delta \mathrm{p}$ is $1.5 \mathrm{Mpa}$.

According to the simulation results, the flow curve of the valve at different opening degrees shown in Fig.9. The calculated results indicate that under the same pressure, the flow gradually increases with the increasing of throttling groove opening degree. When the opening degree is less than $1 \mathrm{~mm}$, the slope of the flow curve is larger, when the opening degree is greater than $1 \mathrm{~mm}$, the flow flat curve. According to the equation (1), the flow coefficient curve can be achieved, as showed in Fig.10, opening degree less than $1.5 \mathrm{~mm}$, the flow coefficient decreased rapidly, with further increasing the opening degree of valve, the flow coefficient gradually increases.

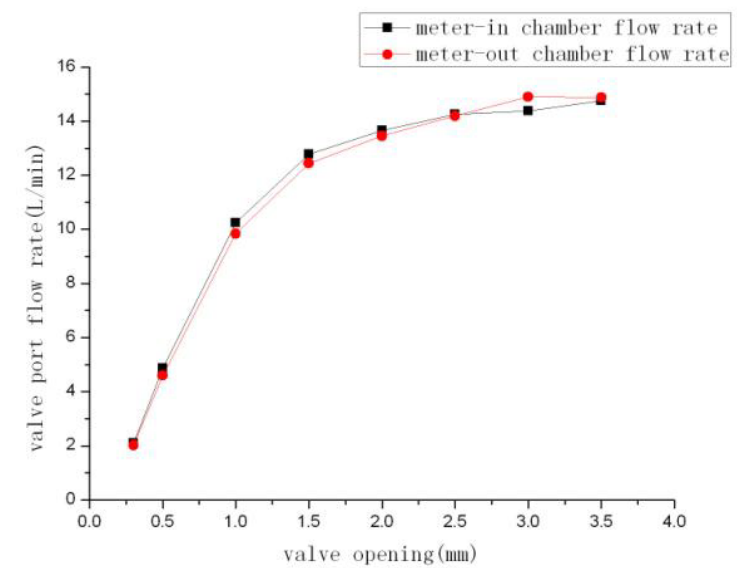

Figure 9. Flow characteristics of the U-shaped slide valve

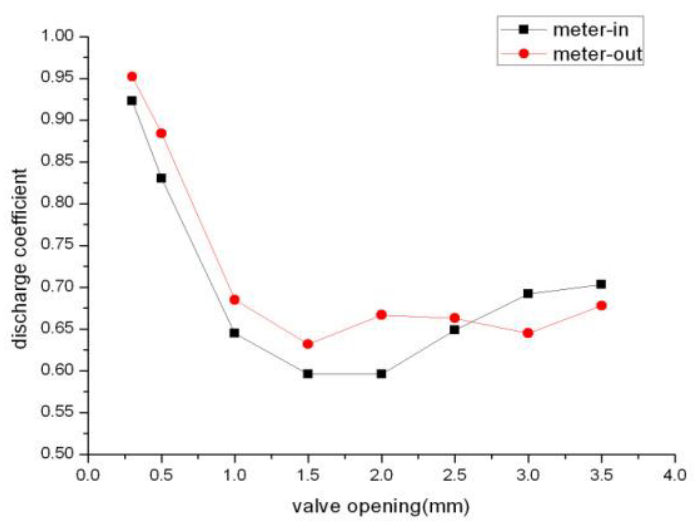

Figure 10. Flow coefficient of the U-shaped slide valve

Due to the effect of the opening degree, the flow coefficient changes instability, thus it can be seen that the flow coefficient is not a stable value. As a result of the flow coefficient curve linearity has a great impact on the stability of the system, it is necessary to use the Fluent software to analysis the flow characteristics and obtained the law of flow coefficient, provide for a reference of slide valve of the optimization design.

\section{Summary}

The theoretical models of U-shaped slide valve was verified by the CFD simulations, in particular, the pressure and velocity distribution have been analyzed here in detail, as it is an important factor contributing to the operation of the valve.

The pressure drop, mainly concentrated in the Ushaped groove, and the vortex was appeared near the outlet oil chamber. With the increase of opening degree, the flow gradually increases.

\section{References}

1. G.Z.Wang, Machine Tool \& Hydraulics. 1(2003) 9596(in Chinese).

2. Q.L.Zhang, Y.Z.Wang, Mechanical and Electrical Equipment. 19(2015) 82-87(in Chinese).

3. J.Zhang, D.L.Gao, X.H.Wang, Chinese Hydraulics and Pneumatics. 4(2015) 56-59(in Chinese).

4.J.Yu, J.Zhuang, D.H.Yu, Chinese Journal of Mechannical Engineering. 27(2014) 1064-1074.

5. C.Samuel M, P.Veerabhardra Rao, Journal of Fluid Engineering, 103(1981) 564-574.

6. H.Ji, X.Fu, H.Y.Yang, Machine Tool \&Hydraulics.5(2003) 14-16(in Chinese). 